\title{
INTERNACIONALIZAÇÃO, TESTES DE PROFICIÊNCIA EFORMAÇÃO DE PROFESSORES
}

\author{
Silvia Regina Gomes MIHO \\ Universidade Federal da Grande Dourados
}

\begin{abstract}
RESUMO: Considerando o programa Idiomas sem Fronteiras - IsF - como uma das principais políticas linguísticas atualmente em vigor no Brasil no que se refere à questão das línguas estrangeiras, fica evidente que sua relação com formação de professores é bastante forte e complexa, por vários motivos, entre os quais ressaltam-se os seguintes: 1) a necessidade urgente de se oferecer proficiência de forma institucionalizada e padronizada para nossos estudantes e pesquisadores universitários; 2) aplicar testes de proficiência aceitos internacionalmente.. Este trabalho almeja discutir a centralidade dos exames de proficiência no programa Idiomas sem Fronteiras (IsF) e sua influência na formação de professores a ele associados. Como abordar os efeitos do 'washback' presentes neste programa no que se refere à formação de professores? Qual o perfil de professores de línguas que queremos formar? Como podemos discutir a questão da proficiência em línguas estrangeiras, mais especificamente em língua inglesa, no atual quadro de internacionalização do ensino superior brasileiro? Após um breve panorama das características internas dos testes e de seus possíveis usos em políticas linguísticas, uma das sugestões feitas por este trabalho é a de que uma visão mais detalhada e compreensiva de aspectos políticos, econômicos e ideológicos que empoderam os testes de proficiência deveriam ser mais diretamente trabalhados e discutidos na formação de professores de línguas.
\end{abstract}

Palavra-chave: testes de proficiência, internacionalização, políticas linguísticas no Brasil

\begin{abstract}
This paper aims to discuss the central role of language proficiency tests in Language without Borders Program (LwB), one influential Brazilian language policy program under implementation. Since the program is directed to improve foreign languages proficiency and apply standard language tests among Brazilian scholars, it is closely related to language teachers' education. To which extent the proficiency tests may influence teacher education within (and also outside) LwB? How should we approach the washback effect on English teacher education? How should we discuss questions involving proficiency for internationalization or academic purposes? What kind of profile do we want for future English language teachers? After a brief overview of the internal features of a proficiency test and its possible uses in language policies, one of the suggestions this paper makes is to that a more comprehensive and detailed view of some of the political, economic and ideological features which empower standardizes tests should be directly addressed in language teacher education.
\end{abstract}

KEY-WORDS: Proficiency tests; internationalization; language policies in Brazil

\section{Globalização e internacionalização: processos e políticas complementares}


Sabe-se que a palavra 'globalização' refere-se a processos econômicos e políticos praticados por vários países no sentido de favorecer e facilitar a mobilidade de capitais, ideias, bens e serviços de maneira mais ágil e eficiente possível. Internacionalização, por sua vez, atua na esfera do ensino superior para ampliar e solidificar os estreitos laços entre produção de conhecimento científico e tecnologia de informação e os mercados internacionais, nas " knowledge-based economies (KBE). Dessa forma, há um sistema que se retroalimenta constantemente. (Altbach, 2016, Knight, 2008). Apesar das múltiplas possibilidades de mobilidade que a globalização e a internacionalização oferecem aos indivíduos, tais processos se efetivam de fato após algumas etapas de processos de avaliação e seleção através de testes padronizados, como, no caso deste estudo, os testes Toefl Itp, produzidos e administrados pela Educational Testing Services (ETS). Aplicados há cinco décadas ao redor do mundo, esses testes foram adotados pelos programas $\mathrm{CsF}$ e IsF como forma de se fazer um diagnóstico da proficiência dos estudantes e pesquisadores brasileiros ao mesmo tempo em que oferecia a oportunidade de certificação internacional aos examinandos que se candidatassem ao programa de mobilidade estudantil. Na grande maioria das universidades ' de excelência' a língua de instrução é a língua inglesa e, mundialmente é necessário que o estudante tenha a certificação de proficiência linguística para poder se candidatar ou inscrever para os programas lá ofertados. O conhecido 'gatekeeping. '

Considerando o que foi exposto, uma reflexão desenvolvida por Ricento (2015) nos serve como ponto de apoio para confirmar a hipótese de que o discurso 'globalizante' afeta como as pessoas pensam e falam sobre línguas; Como o foco está na internacionalização e nos testes de proficiência, tentaremos abordar como o 'discurso globalizante' se atualiza em ações implementadas pelo IsF, seja nos modos como ele se articula com a ciência, principalmente nos documentos oficiais que regem o programa, seja procurando tecer de considerações sobre o teste em si, e de como ele está diretamente ligado às atividades voltadas para a formação de professores dentro do programa.

Evidências textuais de diversas fontes oficiais ou veiculadas pela mídia na divulgação do programa relacionam diretamente vários atributos da globalização (excelência, inovação, competitividade, etc.) à proficiência em língua inglesa. Porém, a proficiência em si e como atingi-la parecem elementos secundários na política de internacionalização do CsF. Primeiro, há a ampla distribuição de bolsas para mobilidade, posteriormente, tomam-se providencias para "capacitar" os bolsistas para as seleções estrangeiras feita via testes de proficiência. Em 2015, foi divulgado o Relatório do Senado Federal do Brasil ${ }^{1}$ sobre o CsF. Referente ao problema da " barreira linguística" enfrentado pelo CsF, o documento declara que as ações do IsF no sentido de melhorar a proficiência linguísticas dos bolsistas CsF foram, originalmente, retroativa|: " A iniciativa foi bem-vinda, mas deveria ter sido criada algum tempo antes do lançamento do CsF." (RELATÓRIO No $\quad$ - CCT, DE 2015, p. 51)

Vejamos, por exemplo, o texto da portaria que institui o IsF/ Inglês de 2013 e o IsF/ Idiomas, de 2014. De seus objetivos, destacaremos os três primeiros.

“Art. $1^{\circ}$ Fica instituído o Programa Inglês sem Fronteiras, com o objetivo de propiciar a formação e capacitação de alunos de graduação das instituições de educação superior para os exames linguísticos exigidos para o ingresso nas universidades anglófonas.

Art. $2^{\circ}$ - São objetivos do Programa Inglês sem Fronteiras:

I - promover, por meio da capacitação na língua inglesa, a formação presencial e virtual de estudantes brasileiros, conferindo-lhes a oportunidade de novas experiências educacionais e profissionais

\footnotetext{
${ }^{1}$ Disponível em: www.senado.leg.br/atividade/rotinas/materia/getTexto.asp?t=184659\&c=PDF...
} 
voltadas para a qualidade, o empreendedorismo, a competitividade e a inovação em áreas prioritárias e estratégicas para o Brasil;

II - ampliar a participação e a mobilidade internacional de estudantes de graduação das instituições de educação superior brasileiras, para o desenvolvimento de projetos de pesquisa, estudos, treinamentos e capacitação em instituições de excelência no exterior;

III - contribuir para o processo de internacionalização das instituições de educação superior e dos centros de pesquisa brasileiros;[...]" ${ }^{2}$

A instituição do programa, justificada por seu primeiro objetivo, evidencia a relação de causalidade que os testes de proficiência exercem sobre a 'formação e capacitação em língua inglesa' e, consequentemente, nas demais ações do programa que daí se originam, inclusive, como declara a Portaria 973, que institui o Idiomas sem Fronteiras (2014) ${ }^{3}$, a inclusão de mais idiomas, além do inglês e a preocupação com a formação de professores, que não estava explícita na primeira portaria. Comparemos os primeiros objetivos dos documentos:

Art. 1o Fica instituído o Programa Idiomas sem Fronteiras com o objetivo de propiciar a formação e a capacitação em idiomas de estudantes, professores e corpo técnico-administrativo das Instituições de Educação Superior Públicas e Privadas - IES e de professores de idiomas da rede pública de educação básica, bem como a formação e a capacitação de estrangeiros em língua portuguesa.

Art. 2o São objetivos do Programa Idiomas sem Fronteiras:

I - promover, por meio da capacitação em diferentes idiomas, a formação presencial e virtual de estudantes, professores e corpo técnico-administrativo das IES e de professores de idiomas da rede pública de educação básica, conferindo-lhes a oportunidade de novas experiências educacionais e profissionais voltadas para a qualidade, $o$ empreendedorismo, a competitividade e a inovação;

II - ampliar a participação e a mobilidade internacional para o desenvolvimento de projetos de pesquisa, estudos, treinamentos e capacitação em instituições de excelência no exterior;

III - contribuir para o processo de internacionalização das IES e dos centros de pesquisa.

Comparativamente, a inclusão de mais categorias de participantes potenciais do projeto expressa a intenção ou a necessidade de se ampliar e se diversificar o público-alvo das ações do IsF. Todavia, alguns dos editais específicos mencionados na Portaria 973 não se publicaram, com destaque aqueles que incluíam os professores da educação básica. Independentemente dos fatores político-econômicos vigentes em 2015 e 2016, os testes de

\footnotetext{
2 PORTARIA № 1.466, DE 18 DE DEZEMBRO DE 2012, disponível no DOU de 19/12/2012.

${ }^{3}$ http://isf.mec.gov.br/ingles/images/pdf/novembro/Portaria_973 Idiomas sem Fronteiras.pdf
} 
proficiência continuam, até o momento, a ação mais abrangente e consistente realizada pelo programa, como os números divulgados pelos gestores do programa em $2016^{4}$ demonstram.

Como, afinal, esses números se relacionam com a formação de professores? De maneira retroativa. Sinteticamente, poderíamos dizer que uma reação em cadeia gerou a urgência na capacitação linguística, o que demanda professores aptos para capacitar para aquele propósito. A comprovação de sua aptidão à função de capacitar e formar estudantes, pesquisadores e professores da rede pública é o seu score (preferencialmente nível $\mathrm{C} 1$ do CEFR) no teste TOEFL ITP, Toefl IbT ou equivalente, além de alguns outros requisitos especificados em editais de cada uma das 63 instituições contempladas com um Núcleo de Idiomas (NucLi), de acordo com as condições operacionais locais.

\section{CEFR, proficiência e testes na formação de professores}

"O programa IsF tem como principal objetivo incentivar o aprendizado de línguas, além de propiciar uma mudança abrangente e estruturante no ensino de idiomas estrangeiros nas universidades do País. " ${ }^{5}$.

Quais mudanças 'abrangentes e estruturantes' podemos inferir das ações do programa até agora, no tocante à formação de professores de inglês, participantes ou não do programa como bolsistas ou alunos? Uma delas, acreditamos, é a aceitação imediata e sem muitos questionamentos dos níveis do CEFR como padrões inquestionáveis de isenção na aferição da proficiência linguística. ${ }^{6}$ Para atuar como professor-bolsista do Isf, deve-se ter score C1. Para ser bolsista IsF, era necessário atingir o nível A2. Deve-se ter experiência com o tipo de linguagem e o tipo de questões do exame para poder capacitar alguém para a mesma tarefa. Mas, qual tipo de proficiência ele testa? Questionar, aqui, não é sinônimo de negar a importância e os processos de validação dos testes, mas sim de se perguntar:

O que legitima tais scores e níveis? Por que eles guiam materiais, métodos e técnicas de ensino? Por que eles se tornam cada dia mais importantes no cenário das universidades e das empresas? É essa a proficiência da qual precisamos, para além do imediatismo que a necessidade impõe, no contexto das bolsas para mobilidade CsF? Essas são questões viscerais que conectam os testes de proficiência e a formação de professores de línguas.

\section{Conceituando proficiência}

Nos estudos linguísticos, termos como proficiência, competência, performance, habilidade são utilizados, muitas vezes como sinônimos, para tratar a respeito das questões originadoras de testes e avaliações: este candidato tem condições de se comunicar na língua alvo? Qual o grau de eficiência que ele consegue atingir em sua comunicação naquela língua?

\footnotetext{
4 “ By April 2016, the LwB Program had 184 test centers in all corners of the country. In this scenario, $1,127,255$ test seats were made available, with 516,434 students registered and 328,766 tests corrected." (Abreu e Lima, D. e Moraes Jr., W., 2016)

${ }^{6}$ As informações e links disponíveis online apresentam de modo bastante superficial o que significa 'nível de proficiência' s, indicando as instituições responsáveis pelo desenvolvimento do quadro comum de referência europeu. http://isf.mec.gov.br/ingles/pt-br/qual-e-meu-nivel-de-proficiencia-em-ingles
} 
John Read (2015) discute o conceito de proficiência, especificamente no campo acadêmico. O autor faz importantes distinções iniciais, fundamentais para entendermos o que os testes significam e como constroem seus significados na área técnica que envolve design, validação e pontuação. Read chama a atenção para o fato de que, antes de se desenvolver e validar um teste, seja qual for sua finalidade, é necessário esclarecer qual é o seu construto subjacente. Construto, no presente contexto, significa o conjunto de parâmetros norteadores dos elementos a serem avaliados num teste de proficiência.

De acordo com Read deve-se estar alerta para o fato de que a natureza subjacente ao construto indica o tipo de conhecimento que os estudantes devem demonstrar nos testes. Indica, também, o conceito de língua, de usos da língua e de concepções ideológicas que inevitavelmente se associam aos testes, principalmente quando eles reproduzem padrões e variedades normatizadas em colocações, gêneros textuais e estratégias discursivas aceitas como adequadas ou 'corretas', os chamados 'standards'. Blommaert (2006, p.512) explica que o 'standard é uma variedade particular de linguagem, percebida como neutra devido a elaborados processos sociais e históricos de normatização e codificação. Agem na construção e reprodução de códigos e gêneros específicos para propósitos públicos, criando um sentido de autoridade que emana de um centro, fazendo de uma forma particular de discurso ser emblemática de pertencimento ao grupo [...]'. No caso específico dos testes TOEFL ITP e das ações dele derivas dentro do programa IsF, o 'standard' padrão é o Native American Speaker com maior ênfase para temas relacionados a assuntos acadêmicos.

Outro fator que não deve ser esquecido é a distinção fundamental entre os conceitos de competência e de proficiência que servem de base para os construtos dos testes. Competência seria definida como o conhecimento sobre o sistema formal da língua, oposto à sua aplicação em situações de comunicação, em performances. Escreve Read que:

It should be emphasized that competence does not refer just to explicit knowledge of grammatical terms and concepts or the meanings of words; it primarily covers the implicit knowledge that is acquired by native speakers and underlies their intuitions about what is correct and appropriate. (Read, 2015, p. 80)

Além do mencionado ' conhecimento implícito' de falantes nativos, os testes também avaliam a 'competência estratégica' no uso da língua, ou seja, o 'uso funcional da linguagem para fins comunicativos.' (Read, 2015, p.80). Em sua maioria, os assim chamados testes de proficiência são caracterizados por avaliar o desempenho do examinando no que se refere à sua competência estratégica de comunicação na língua alvo num contexto específico, e usando-se instrumentos específicos de avaliação

O conceito de 'proficiência' presente no TOEFL ITP, utilizado pelo programa IsF desde 2014 em larga escala, reflete, tanto em seu construto quanto em seu conteúdo, que o teste permanece sem modificações significativas em sua estrutura desde seu início, na década de 1960, conforme indicam as leituras de Chapelle et al (2008) e Read (2015). A verificação deste fato nos leva a pensar sobre a adequação desta estrutura de testes ao contexto atual, no que se refere à capacitação que o programa oferece aos seus participantes, dado o longo período desde seu lançamento no mercado de mundial de testes. Outro questionamento pode ser dirigido à falta de atenção às habilidades produtivas no teste TOEFL ItP, que pode gerar uma noção de proficiência parcial e precária, já que o contexto específico do teste só avalia habilidades receptivas.

No que se refere ao 'conhecimento implícito' de falante nativo que o teste avalia, podese dizer que ele reforça estereótipos, preconceitos e o ' native speakerism', em detrimento de 
abordagens mais plurais, de tendências não hegemônicas. Este tipo de conhecimento implícito e os valores que ele traz consigo tem relação direta com a formação de professores de línguas. A valorização deste conhecimento está conectada a questões de autoridade e de poder através de processos de 'construção e reprodução de gêneros e códigos para propósitos públicos, criando um sentido de autoridade que emana de um centro, marcando certas formas de falar como emblemáticas de grupos e identidades'. Blommaert (2006, p.511).

Ainda em relação ao fato do conteúdo do teste se manter praticamente inalterado por cinquenta anos, gostaria de interpretá-lo a partir da observação feita por Hymmes de que 'enquanto as formas linguísticas podem permanecer estáveis através de diferentes contextos, situações e grupos de usuários, o seu valor indicial pode diferir drasticamente. ' (citado por Blommaert, 2006, p.512). Em outras palavras: o teste continua medindo partes estáveis e controláveis da língua, relacionadas ao sistema linguístico, que permanecem sem alterações formais, porém, o valor indicial de formas linguísticas transforma-se, refletindo nas expressões e construções linguísticas escalas de valores extralinguísticos: aspectos políticoeconômicos que dão origem às desigualdades sociais são marcados linguisticamente, sendo, na maioria das vezes, percebido como hierarquicamente inferiores à linguagem considerada 'padrão'.

\section{Testes de proficiência}

Devido à importância que os testes de proficiência vêm recebendo por parte de autoridades políticas e econômicas nas últimas décadas, vários aspectos da elaboração dos mesmos sofreram transformações que, de certa forma, atuaram como respostas às pressões de seu contexto de origem. McNamara (20.., p,40) explica que anteriormente os testes eram desenvolvidos com base em teorias da proficiência linguística e da comunicação para elaborar construtos que evidenciariam informações relevantes para os objetivos e contexto de aplicação de um teste em particular. Outra mudança ocorrida nas últimas cinco décadas está no modo como são pontuados e computados os resultados dos testes. Testes padronizados em larga escala, como Ielts e Toefl, por exemplo, precisam de justificação teórica e aplicabilidade eficiente nos mais variados países e culturas. É um processo de abstração, generalização e comparação de resultados sem referência direta ao seu contexto educacional imediato. O score final depende de um processo de inferência.

Fulcher (2013) elenca duas orientações distintas para o uso de testes. A primeira, seria uma necessidade ou orientação inerente ao processo educativo, (internal mandate) de caráter formativo e processual. A segunda, seguindo orientações externas (external mandate), tende a avaliar de modo a 'medir a proficiência do aprendiz sem referência ao contexto', geralmente, aplicados em larga escala e também considerados de 'alto risco' (High stake tests). Read (2015) sugere tipos diferenciados de testes: os testes diagnósticos, os testes de nivelamento e os testes de proficiência, embora ressalte que os termos são usados como sinônimos na maioria dos estudos realizados sobre o tema.

Os testes formativos ou processuais, são necessários em práticas educativas., assim como os testes diagnósticos, uma vez que estes têm como principal meta identificar pontos fracos do candidato/aprendiz para futuras intervenções pedagógicas, apropriadas às suas necessidades, conforme explica Alderson (2005). 
Quanto às principais características dos testes de proficiência com orientações vindas de fora do contexto educacional imediato, Fulcher (2010) lista as seguintes: generalização, testes padronizados e rituais. Podemos mencionar como exemplos os testes TOEFL e IELTS ${ }^{7}$.

Como se sabe, esses testes se pautam pelo CEFR. De acordo com Fulcher (2010) essa relação se estabelece e se justifica na argumentação envolvida na validação dos testes e nos processos inferenciais necessários para que a relação se mantenha e se retroalimente.

The assumption that the CEFR scales have been constructed on a principled analysis of language use within a range of domains, or a theory of second language acquisition, is mistaken. The scale descriptors were drawn from existing scales in many different testing systems from around the world, and were placed within the CEFR scales because teacher judgments of their difficulty could be scaled using multi-faceted Rasch (North 1996). (p.16)

A questão agora é: como são elaborados os 'descritores' (descriptors) que caracterizam as escalas/níveis de proficiência (A1, A2, etc.)? Fulcher (2010, p.18) afirma que:

The selection of descriptors for the CEFR scales, and scale assembly, was psychometrically driven; or as North (1995) says, based entirely on a theory of measurement. The data in the scaling studies were intuitive teacher judgments rather than samples of performance. What we see in the CEFR scales is therefore "essentially a-theoretical" (Fulcher 2003: 112), a critique which North and Schneider (1998: 242- 243) admit to be the case. Since this analysis, it has been frequently repeated that the scales have no basis in theory or SLA research (Hulstijn 2007: 666).

As escalas, por sua vez, são derivadas de padrões estatísticos e psicométricos unidimensionais e que não têm nenhum paralelo ou semelhança entre si, pois tratam de aspectos distintos de um mesmo objeto: as estatísticas são lineares por natureza e função, enquanto a aprendizagem de línguas, um processo cognitivo, também tem suas escalas avaliativas unidimensionais, porém também considera outros aspectos além dos resultados apresentados por testes padronizados de múltipla escolha

Ao analisar os descritores e as escalas do CEFR, Fulcher (2010) questiona o modo como as habilidades por eles elencadas foram organizadas, ressaltando, como a própria orientação do Conselho europeu o faz, que o contexto não é levado em consideração para poder acomodar resultados generalizados a partir de uma mesma escala. Segundo ele, o contexto de interação é uma lista não estruturada, os descritores na escala não representam uma análise linguística ou discursiva da língua alvo. Os descritores são organizados do modo como são simplesmente pelo fato de que a percepção de sua dificuldade é suscetível à manipulação estatística.

Restam, ainda, dois termos importantes para esclarecermos: estrutura (framework) e modelo (model). Para Fulcher, estrutura é o que dá sustentação teórica ao construto em um contexto específico, capaz de gerar especificações, enquanto modelo pode ser definido como “ fonte de ideias para a seleção de construtos que são úteis e relevantes para a elaboração de testes para fins específicos." 8 O CEFR é um modelo e não uma estrutura. Testes de

\footnotetext{
${ }^{7}$ Cf,.Shohamy (2014, pp 61-67) Para uma descrição completa das características deste tipo de teste

8 In effect-driven testing the purpose of a model is to act as a source of ideas for the selection of constructs that are useful and relevant to the design of tests for specific purposes. (Fulcher, 2010,p.18)
} 
proficiência específicos, são estruturas. Por esta razão, estabelecer ligações entre a pontuação de testes e as escalas do CEFR é um equívoco, já que estrutura e modelo são duas estâncias diferentes da arquitetura documental dos testes. A terceira delas são as especificações.

Gostaria de ressaltar duas observações de Fulcher (2010) no que se refere ao CEFR e sua relação direta com o Conselho Europeu e com a OECD. Sobre o Conselho Europeu:

1) "The Council of Europe Recommendation states that CEFR( Council of Europe, 2001) is " purely descriptive - not prescriptive, nor normative." And says that its use should be selective, to promote "social inclusion, intercultural dialogue, active democratic citizenship, language diversity, plurilingualism, learner autonomy and lifelong learning. "It is difficult to disagree with the idealism behind such language. However, the recommendation itself is for governments to adopt the CEFR as the key tool in linguistic policy [...] that it becomes the basis for language teacher training, the organization of coursebooks and curricula, the composition of educational institutions, and the recognition of language qualifications. " (p.14)

O autor ainda ressalta que a OECD e o Conselho Europeu tem objetivos comuns, principalmente durane o período que antecede a implemenação do CEFR e da consolidação da globalização em território europeu. Outro fato que não pode ser deixado de lado é a relação das palavras do construto com o conteúdo do teste, sua validação e usos que dele se faça, pois é a partir do construto que a própria política se origina. De modo suscinto, McNamara relaciona " os termos nos quais os resultados da educação linguística são especificados, e que são o construto da avaliação linguística são expressões de política. E as formulações de certas estruturas, incluindo o modo como elas se prestam à redução a simples números - A1, A2, etc - são desenhados para satisfazer as necessidades daqueles responsáveis pela governança dos sistemas educacionais."

É interessante observar que as recomendações do conselho europeu estão em relação direta com políticas linguísticas europeias e globais, o que influencia na adequação de construtos e de validação dos testes, desencadeando a produção, consumo e uso de toda uma gama de produtos e serviços educacionais em função dos testes e de suas orientações políticoeconômicas.

Fulcher (2010) acrescenta que:

At the supra national level one example of the use of a system that is increasingly being used to harmonize and control language learning, to deal with perceived threats such as a weakened position in global markets, is the Common European Frame of Reference (CEFR) (Council of Europe, 2001; Fulcher, 2004). Its primary use is emerging as a tool for designing curricula, reporting both standards and outcomes on its scales, for recognition of language qualifications through linking tests scores to levels on the CEFR scales. [...] This is closely tied to the implementation of the Bologna declaration (1999) to harmonize and standardize higher education, including structure and content of programs. (2010, p.8).

Complementando, Shohamy (2006, p.85) indica outros aspectos a serem levados em consideração quando testes são escolhidos como o principal instrumento de uma política e de 
um planejamento linguístico: testes são vistos como instrumentos de autoridade pelo público; mostram ação por parte das instituições responsáveis; permitem uma pontuação flexível. Como a autora explica, testes de proficiência podem ser mecanismos velados de manutenção e controle de relações de poder que se estabelecem através das línguas, pautando seu ensino, sua aprendizagem, sua avaliação e seus usos.

Menken (2008) discute vários aspectos da política educacional norte-americana $N o$ Child Left Behind (NCLB), entre eles, os que mais se assemelham ao contexto de implementação do IsF estão relacionados à aplicação de testes padronizados como fator primordial de todas as ações decorrentes da política linguística, ligando diretamente os testes e a sua administração contábil/administrativa (accountability). Afirma Menken (2008, p.181) que esta política se caracteriza, principalmente, por aumentar o envolvimento federal na educação pública, por ser uma política descendente (top-down); por ser centralizadora “ amarrando o financiamento ao Departamento Federal de Educação, para garantir que os mandatos sejam seguidos e por ter uma real implementação em sala de aula.

Desse modo, podemos inferir que o programa IsF partilha com o NCLB os três elementos acima descritos. Embora consideremos que o NCLB se dirige ao público de estudantes norte-americanos que passarão pelos exames ao final do ensino médio (high school) e, portanto, mira seu foco para dentro do país em que se implementa enquanto que o IsF volta seus objetivos na internacionalização, os mecanismos utilizados pelas duas políticas se assemelham de forma clara, assim como também convergem no sentido de uma homogeneização da língua padrão e de sua manutenção como instrumento de exercício de poder. Os testes padronizados ampliam o abismo entre a linguagem padrão e os mais diversos usos da linguagem na comunicação cotidiana. Os procedimentos e instrumentos dos testes padronizados medem resultados, sem relacioná-los nem ao contexto do examinando e nem ao progresso da aprendizagem, confirmando a primazia da 'prestação de contas' sobre a aprendizagem de ambos os programas.

Outro aspecto relevante para a discussão sobre a relevância dos testes de proficiência não apenas na internacionalização, mas também no processo educativo como um todo, está no fato de que pouco se discute a respeito da interpretação e dos usos dos resultados dos testes. O’Loughlin (2013), argumenta, apoiado em Spolsky (2008), que tanto púbico em geral quanto administradores educacionais têm uma visão muito simplificada sobre a produção, interpretação e usos dos testes de proficiência. Trata-se de escalas unidimensionais insuficientes para avaliar a dinamicidade que é, de fato, a proficiência. $\mathrm{O}$ autor também alerta para os problemas que uma política educacional baseada no uso de testes pode acarretar, dentre eles, o mais evidente é a crescente comoditização dos scores de testes de proficiência em um mercado educacional altamente competitivo, que busca alunos internacionais, cujas anuidades e outras tarifas serão parte fundamental da geração de lucros para as universidades anglófonas ou que adotam o English Medium of Instruction (EMI).

\section{Formação de professores e o ' déficit model'}

[...] Tests are de facto policy in schools and essentially become policy for language education when curriculum and teaching are aligned to tests. Testing and accountability under the law ultimately reflect a 'language problem' or a 'deficit model' orientation, where language 
has become a liability for the English language learner. (Menken, 2008, p. 169)

A formação de professores de língua inglesa no Brasil contemporâneo precisa ser pensada e alguns aspectos que até o presente o momento não tinham tanta visibilidade precisam ser trazidos para a discussão, como, por exemplo, estudos que situem as línguas, seus estudos, ensino e aprendizagem num contexto social, político e econômico mais amplo que a metalinguagem dos estudos linguísticos, dialogando mais diretamente com as forças ideológicas e econômicas que, mesmo de forma velada, regem as práticas dos professores em sala de aula, como ocorre com os níveis de proficiência do CEFR.

É bastante comum o estudo de metodologias e técnicas de ensino de línguas, aspectos culturais e sociais, mas pouco frequente, na maioria dos cursos de graduação do país, é a presença de espaços acadêmicos que discutam como e porque somos pautados pelos níveis do CEFR, o que aqueles scores realmente significam, qual tipo de proficiência os testes medem e por que devemos aceitar (ou não) sua autoridade. Acreditamos que professores e, principalmente, futuros professores devem entender claramente esses pontos, caso contrário estarão atuando na reprodução de valores, padrões e ideologias hegemônicos de modo quase automático. E isso é um fato preocupante para o campo da formação docente.

Quando uma política linguística, como aquela do IsF, é implementada e celebrada por seus avanços e conquistas, não se pode esquecer do caráter retroativo e de remediação dessa política e de como ela se insere nas relações de internacionalização e formação de professores de línguas. Concordamos com Canagarajah (citado por Block 2015, p.385) quando afirma que o mesmo sistema de monopólio de produtos industriais que os países economicamente mais desenvolvidos impõem aos países em condições menos favoráveis se reproduz no campo do ensino de língua inglesa, através de seu mercado de materiais e métodos, concepções, discursos que revestem ideologias de grupos específicos.

Os testes Toefl são um ícone deste mercado e desse monopólio dos países do 'Inner circle'. Políticas e esforços são feitos para que se possa passar pelo gatekeeping de modo rápido e, assim, aproximar-se das experiências educacionais no exterior. Trata-se de uma política de acomodação (Park e Wee, 2008) e não de apropriação ou ressignificação. Seria essa uma direção interessante ou, pelo menos, vantajosa, para se seguir na formação docente, visando a internacionalização?

Acreditamos que a importância da língua inglesa e dos testes de proficiência no mundo contemporâneo se acentua com o desenrolar de processos político-econômicos nos quais a fluência naquela língua é fundamental. E, por essa mesma razão, os aspectos políticos e econômicos relacionados a ela poderiam ser mais explorados na formação de professores.

Blomaert e Dong (2015) afirmam que há dois paradigmas em curso nos estudos da linguagem. O primeiro, mais tradicional, recorta línguas e as isola para fins de estudo e análise. É a partir desse primeiro paradigma que testes como o TOEFL ItP retiram suas estruturas e seus construtos. Porém, o convívio com as transformações constantes torna mais produtivo e proativo um segundo paradigma, que os autores assim descrevem:

The second paradigm can be called a sociolinguistics of mobility, and it focuses not on language-in-place but on language-in-motion, with various spatio-temporal frames interacting with one another. Such spatio-temporal frames have been described as 'scales,' and the assumption is that, in an age of globalization, language patterns must be understood as patterns that are organized at different, layered scalelevels (Blommaert 2005, 2007; Collins 2007). Access to, and control 
over, scales is unevenly distributed; it is a matter of power and inequality - as becomes clear when we consider typical resources for access to higher scales (that is, non-local and non-situationally specific ones) such as a sophisticated standard language variety, or advanced multi-modal and multilingual literacy skills."

Adotar uma formação de professores voltada para testes de proficiência para resolver problemas educacionais que há décadas se arrastam sem solução pode ser uma política de fácil e rápida implementação e controle, mas é parcial e paliativa. Além de passar pelo gatekeeping, a formação de professores de línguas poderia auxiliar não apenas no acesso às escalas e níveis estipulados como 'gatekeepers' pelos testes e editais, mas, acima de tudo, favorecer o questionamento e a discussão para que o 'controle' sobre essas escalas sejam melhores distribuídos. Uma concepção de língua como recurso - como capital simbólico e cultural, além de econômico, ao qual se agregam valores - poderia substituir uma concepção tecnicista que entende línguas, suas diversidades e possibilidades como problemas a serem resolvidos, buscando a padronização e a homogeneidade. Nesse contexto, a formação do docente de língua estrangeira se encontra numa situação constante de tensão entre ' as formas relativamente anônimas, padronizadas, sem agentes e transferíveis de padronização e formas identificáveis de autenticidade.' (Heller, 2015, p.471).

Park e Wee (2008) sintetizam o processo de comoditização da linguagem de maneira clara. Segundo os autores, esse processo envolve a transformação da ideia de linguagem enquanto forma de marcar a identidade social de alguém e que, portanto, não está sujeita a troca e linguagem como recurso econômico que pode ser cultivado para obtenção de lucro ou ' adquirida como uma habilidade que pode ser oferecida ao mercado.' (p.125)

Como a formação de professores discute, atualmente, a 'comoditização da linguagem' e o papel dos testes de proficiência no contexto atual? A transformação da linguagem em produto? Este, talvez seja um dos pontos mais importantes na formação docente de linguagens, como língua portuguesa, inglesa, etc. e, também, de docentes de linguagem matemática e científica, nas economias do conhecimento. Não podemos nos esquecer de que as economias do conhecimento produzem, registram, divulgam o conhecimento quase que exclusivamente em língua inglesa. Ou seja, os processos político-econômicos de produção, circulação e distribuição do conhecimento e da tecnologia em curso estão sujeitos, segundo Llantada (2012), às pressões pela mercantilização discurso científico, disponibilizando-o também como mais um produto a ser adquirido e que foi produzido a partir de conceitos comumente aceitos nas sociedades neoliberais: como prestação de contas (accountability), aplicabilidade e capacidade de transferência do conhecimento. Consegue acesso e manutenção no poder o grupo de forças que controlar, das várias formas possíveis, os usos da língua inglesa e seus discursos.científico-globalizantes, nas diversas instâncias e camadas que compõem as relações entre pessoas, instituições e economias no início do século XXI.

Encerro estas reflexões com as palavras de Park e Wee, por concordar com sua proposta e achar possível encontrar, num futuro breve, mais um caminho na formação de professores de línguas.

"what we need is to find a way to promote a view of standards that is grounded on a more reflexive and critical standpoint [...] to provide ways through which members of a society may more openly engage in critically questioning the indexical processes by which standards come to be valuable." 


\section{Referências}

ABREU LIMA, D. M. E MORAES FILHO, W.M. Languages without borders program: building a Brazilian policy for teaching languages towards internationalization. In: FINARDI, K.R. (org.). English in Brazil: views, policies and programs. Londrina: Eduel, 2016, 97-124

ALDERSON, C.J. Diagnosing foreign language proficiency: interface between learning and assessment. Continuun Books: New York, 2005.

ALTBACH, P. Global perspectives in higher education. Baltimore: Johns Hopkins, 2016

BLOCK, D. Globalization and Language teaching. In: COPELAND, N. Handbook of Language and Globalization. UK: Wiley \& Sons, 2013, 377-402

BLOMMAERT, J. Language Ideology. In: BROWN, K. (editor-in-chief). Encyclopedia of language and linguistics. Second Edition, vol. 6, 510-522. Oxford: Elsevier, 2006.

BLOMMAERT, J E DONG, J. Language and movement in space. In: COPELAND, N. Handbook of Language and Globalization. UK: Wiley \& Sons, 2013. 498-515

CHAPELLE, A. et al. Building a Validity Argument for the Test of English as Foreign Language. New York: Routledge, 2008.

COMISSÃO DE CIÊNCIA, TECNOLOGIA, INOVAÇÃO, COMUNICAÇÃO E INFORMÁTICA. AVALIAÇÃO DE POLÍTICAS PÚBLICAS PROGRAMA CIÊNCIA SEM FRONTEIRAS. Disponível em: www.senado.leg.br/atividade/rotinas/materia/getTexto.asp? $\mathrm{t}=184659$ \&c $=$ PDF...

FULCHER, G. Test use and political philosophy." Annual Review of Applied Linguistics, 2009: 3-20.

FULCHER, G. "The reification of the Common European Framework of Reference." Edição: Greek Association of Applied Linguistics. 14th International Conference of applied Linguistics. Thessaloniki, 2010.

FULCHER, G. Practical Language Testing. New York: Routledge, 2013.

FULCHER, G. Re-examining language testing: philosophical and social inquiry. New York: ROUTLEDGE, 2015.

HELLER, M. Language as a resource in the globalized new economy. In: COPELAND, N. (ed). Handbook of Language and Globalization. UK: Wiley \& Sons, 2013, 471-497

LIANTADA, C. Scientific Discourse and the Rhetoric of Globalization The Impact of Culture and Language. London/New York: Continuum International Publishing Co., 2012

McNAMARA, T. e SHOHAMY, E. Language Tests and Human Rights. International Journal of Applied Linguistics, 2008: 89-95.

McNAMARA, T. "Managing Learning: Authority and Language Assessment." In: Global Perspectives, Local Initiatives, 39-51. 2009.

"The use of language tests in the service of policy: issues od validity." Revue française de linguistique appliquée , 2010: 7-23.

MEC, Ministério da Educação -. Ciência sem fronteiras. 28 de 09 de 2016. http://www.cienciasemfronteiras.gov.br/web/csf/o-programa.. Acesso em 10/12/2016 
MENKEN, K. English learners left behind. Standardized testing as language policy. Clevendon/Buffalo/Toronto: Multilingual Matters, 2008.

O'LOUGHLIN, K. Developing the assessment literacy of university proficiency test users. Language testing, 30(3) 363-380, 2013. 1tj.sagepub.com/

PARK, J.S. e WEE, L. Markets of English. Linguistic Capital and Language Policy in a globalizing world. New York/London: Routledge, 2012

PORTARIA

NORMATIVA

de

http://www.cmconsultoria.com.br/imagens/diretorios/diretorio16/arquivo4359.pdf . Acesso: $10 / 12 / 2016$

READ, J. Assessing English Proficiency for University Study. London/New York: Palgrave MacMillan, 2015.

RICENTO, T. Language policy and Globalization. in: COPELAND, N. (ed). Handbook of Language and Globalization. UK: Wiley \& Sons, 2013,198-209

SHOHAMY, E. Language Policy: Hidden agendas and new approaches. New York: Routledge, 2006

The Power of Tests. A critical perspective on the uses of language tests. New York: Routledge, 2014. 